\title{
NUESTRO OBJETIVO: SEGUIR ACTIVAS
}

\author{
Isabel Labuiga Tomás
}

montredo@hotmail.com

\begin{abstract}
Recepción Artículo: 27 agosto 2021
Admisión Evaluación: 07 septiembre 2021

Informe Evaluador 1: 12 septiembre 2021

Informe Evaluador 2: 13 septiembre 2021

Aprobación Publicación: 15 septiembre 2021
\end{abstract}

\section{RESUMEN}

La relación e intercambio de intereses y conocimientos de un grupo de mujeres que forman una asociación desde hace 28 años, con el objetivo de ser ciudadanas activas, emporedadas, una masa crítica y con acceso a recursos educativos y culturales. De pronto, sorprendidas por una pandemia, el COVI-19, les obliga a bloquear su actividad, provoca en ellas ansiedades y miedos y, les recuerda la vulnerabilidad del ser humano.

Esta situación, nos hace reprogramar de nuevo sesiones ya planteadas que les ayuden en el reencuentro a preguntarse, el por qué somos una asociación de mujeres y que la responsabilidad como tal, nos dirige hacia un envejecimiento activo en el que nos van a surgir problemas que pueden abarcar el área de la salud, el área psicosocial y la psicológica.

Hemos de tener en cuenta que: "Dado que el desarrollo del ser humano dura mientras dura la vida, también la vejez puede ser una fuente de crecimiento personal y aprendizaje"

R. Fernández-Ballesteros. (pág. 40).

Palabras clave: responsabilidad; envejecimiento; salud; participación

\section{ABSTRACT}

Our goal: to stay active. The relationship and exchange of interests and knowledge of a group of women who have formed an association for 28 years, with the aim of being active, cobbled citizens, a critical mass and with access to educational and cultural resources. Suddenly, surprised by a pandemic, COVID-19, which forces them to block their activity, causes them anxieties and fears and reminds them of the vulnerability of the human being.

This situation makes us reschedule again sessions already raised that help them in the reunion to ask themselves why we are an association of women and that the responsibility as such, directs us towards an active aging in which we will arise problems that can cover the area of health, psychosocial and psychological area.

We must bear in mind that: "Since the development of the human being lasts while life lasts, old age can also be a source of personal growth and learning" R. Fernández-Ballesteros. (p. 40).

Keywords: responsibility; aging; health; participation 


\section{NUESTRO OBJETIVO: SEGUIR ACTIVAS}

\section{INTRODUCCIÓN}

Las Naciones Unidas (ONU) refiriéndose a la creación de espacios sostenibles, espacios verdes y oportunidades durante la vejez, redactó la Agenda 2030 con la que incidir en las políticas creadoras de unas sociedades más inclusivas. También la Organización Mundial de la salud (OMS) decretó la década 2020-2030 como del "Envejecimiento saludable". Estas iniciativas implican un compromiso político y de ciudadanía, con derechos y responsabilidades. Derechos que los responsables políticos tienen que legislar para su puesta en práctica, pero, también la ciudadanía, somos responsables en nuestro proceso de envejecimiento de poner en acción nuestra responsabilidad según los principios extraídos de C.D. Hyver y L.M. Gutiérrez Robledo (2013):

\section{Responsabilidades:}

Las personas de edad tienen la responsabilidad de:

Permanecer activas, alertas, capaces, confiadas en el esfuerzo y útiles.

Aprender y aplicar principios sanos de salud física y mental a su propia vida.

Hacer planes y prepararse para la vejez y la jubilación y aprovechar la enseñanza encaminada a la alfabetización.

Adaptarse a los cambios que con el tiempo puedan ocurrir en las relaciones familiares.

Actualizar sus conocimientos y su adiestramiento, si fuera necesario, para aumentar sus posibilidades de obtener empleo si desean participar en la fuerza del trabajo.

Poner a disposición de los demás, en particular de las generaciones más jóvenes sus conocimientos, aptitudes y valores espirituales.

Participar lo más plenamente posible en la vida cívica de su sociedad.

Buscar y desarrollar posibilidades de prestar servicios a los menos afortunados.

Dar a conocer a su médico y sus seres queridos su voluntad respecto de los cuidados que quieren que se les dispense en la última fase de una enfermedad incurable.

Por ello, en nuestro taller intentamos hacer una revisión teórica sobre el "Envejecimiento activo" que concluiremos con una puesta en práctica en un contexto concreto que representa al 52\% de la población mundial.

Ya que como demuestra el Instituto Nacional de Estadística (INE), el tanto por cien de personas de edad, son mujeres, estos datos indican una feminización de la vejez.

Fig.1.Diferencia entre la población de hombres y mujeres por grupo de edad ,2019 (INE)

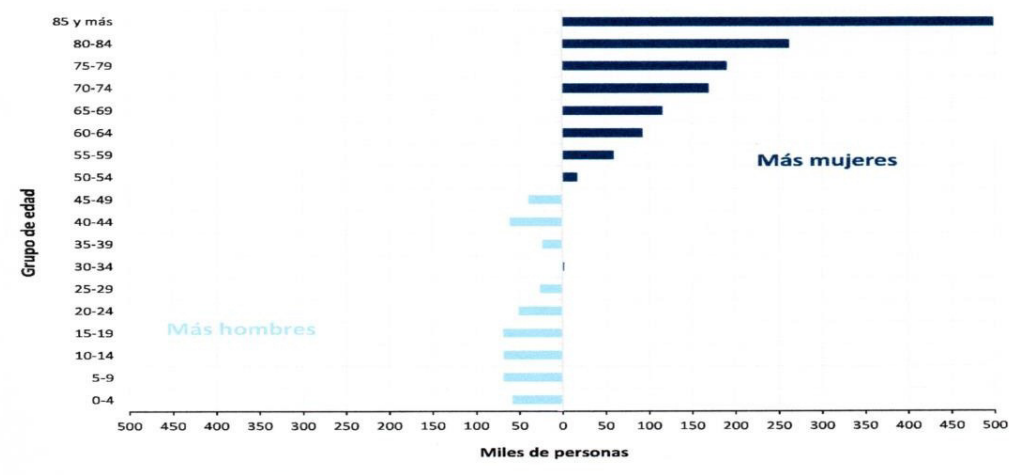

Fuente: INE: Estadística del Padrón continuo a 1-1-2019. Consulta enero 2020. 
Un mundo globalizado como el nuestro, aunque, no en todas las sociedades, rurales 0 urbanas, de diversas culturas 0 en hombres y mujeres, se vive de igual forma la vejez. A pesar de que el S.XX ha aportado tres aspectos a destacar: a) la revolución de la longevidad, cada vez vivimos más años, b) el control de la natalidad y, c) la incorporación de la mujer al mundo laboral. Estos aspectos suponen para las mujeres el tener una conciencia de libertad porque, cuando los hijos/as abandonan el "nido" disponemos de nuestra soledad en libertad y podemos obtener beneficios económicos por nuestro trabajo, ganamos en independencia. Además, el control de la natalidad, nos aparta de la dependencia de la reproducción y nos ayuda a buscar el placer

Fig.2. Aportaciones del siglo XX. Elaborado a partir de Freixas (2006)

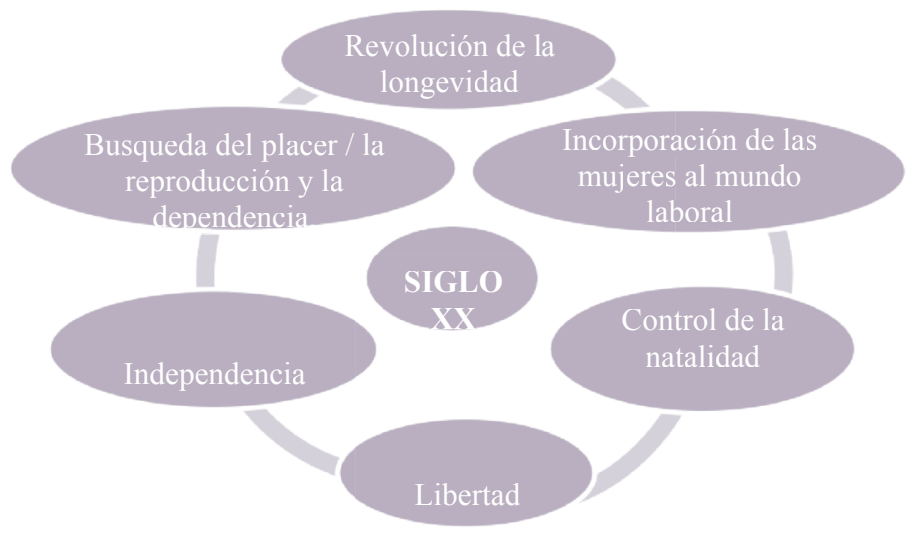

Pero estos logros no han supuesto la desaparición de estereotipos y acepciones sociales con un carácter negativo sobre el progreso del S.XX, como por ejemplo la cosificación de la mujer observada desde su "yo físico" desde fuera o, el "edadismo" con aquello de "pero si yo ... ya" también la pérdida del "ser femenino" por la menopausia y algo que a menudo nos encontramos, el desconocimiento del mundo sanitario sobre la vida de las mujeres que asocian sus problemas físicos a la "ansiedad + hipocondría". Todo ello condiciona a cierto número de ellas, a "hacerse pasar" por más joven y no asumir que envejecer es una nueva oportunidad.

\section{OBJETIVO}

El progresar con estos planteamientos sigue siendo un tanto difícil, la presión social sigue y ante ciertas situaciones detiene el camino empezado. Por ello el objetivo principal para este grupo de mujeres emporedadas, se hace imprescindible el volver a reflexionar sobre dichos logros.

Objetivo: seguir activas. De acuerdo con el principio feminista "hay mucha libertad disponible".

Romper el bloqueo de la pandemia del COVID-19 y en la medida de lo posible, volver a la programación de las actividades habituales de la asociación recordando aquellos principios que la iniciaron.

\section{PARTICIPANTES}

La programación del taller está prevista para un grupo de mujeres $\geq 60$ años, algunas jubiladas, casadas, separadas, procedentes de diversas profesiones, amas de casa y de diversas comunidades o creencias. Todas sin exclusión participan en actividades formativas o de ciudadanía, pero, en estos momentos les paraliza el miedo ante lo desconocido de la pandemia, por ellas y por sus familiares y amigos/as, su capital social que forman parte de su red de cuidados. 


\section{METODOLOGÍA}

Se basa en la definición que sobre el envejecimiento activo hace la OMS: proceso de optimización de las oportunidades de la salud, participación y seguridad con el fin de mejorar la calidad de vida a medida que las personas envejecen.

Fig.3. Pilares del envejecimiento. Elaboración propia a partir de, Martin Lesende, (2008)

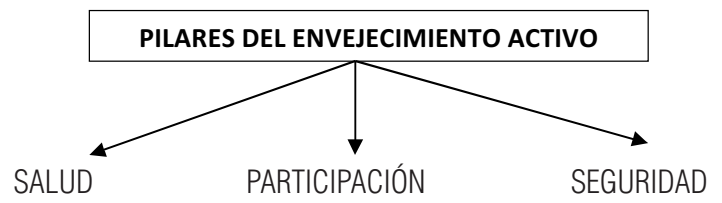

La programación va a seguir la rutina ya conocida: charlas coloquio y talleres (Anexo.1), así como alguna visita 0 excursión. Talleres con una asistencia más reducida porque van dirigidos hacia intereses más personales. El que presentamos en este trabajo va a estar centrado en las charlas coloquio.

Antes de concretar la programación, hay prevista una reunión con las ponentes especialistas de cada tema, con la finalidad de unificar criterios por parte del grupo de la asociación encargado de la puesta en marcha, y del grupo de ponentes. Como especialistas cuentan con la suficiente preparación; además, conocen la asociación y saben de las dificultades para de nuevo, ponernos en marcha.

\section{1ª Sesión:}

Presentar el tema monográfico "Envejecimiento activo" para su aprobación por la asamblea de socias.

Temas: - Asociacionismo

-Participación ciudadana

-Escribir

-Salud fisiológica: Ejercicio

El 2ํㅡㄹércoles de cada mes Nutrición

Caídas

-Salud psicológica

Responder a un cuestionario sobre las responsabilidades de las personas de edad, para tener una base de lo que opina el grupo de mujeres. (Anexo 2).

\section{2a Sesión:}

Tema: La Iongevidad es un triunfo histórico, porque nos ofrece: Ponente: Aportación teórica sobre el "Envejecimiento activo"

Fig.4.Nuevos envejecimientos. A partir de Bateson, M.C. (2011)

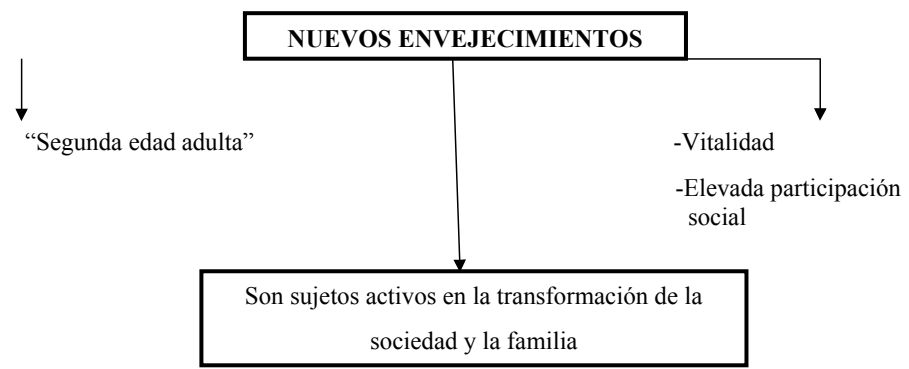


Porque, además, siguiendo las recomendaciones de la Unesco para el S XXI, hemos de aprender a ser mayor y eso requiere pensar en nosotras, aprender a ser egoístas. A olvidarnos del "yo...ya" y seguir haciendo. Tener un crecimiento personal y de aprendizaje y, a vivir en relación con las nuevas generaciones.

\section{3esión:}

Tema: El asociacionismo.

Ponente: Profesora autora de una tesis doctoral sobre asociaciones.

¿Por qué? Porque "El ser independientes de la familia, tener amplias redes sociales, preocuparse por los demás (por la familia, por los amigos y por otros colectivos) y trabajar por ellos es una forma de no sentirse solo y envejecer bien" (Fernández-Ballesteros,2002. Vol.1pag.51).

Como propuesta el cuestionario sobre las relaciones sociales. (Anexo 3).

La asociación nos facilita una relación e intercambio del tiempo y de intereses y saberes

Es una masa crítica de gran valor

\section{4a Sesión:}

Tema: Participación ciudadana.

Ponente: Autora de varios escritos sobre movimientos de participación ciudadana.

En el S.XX el objetivo era sacar a la mujer del ámbito doméstico y, ahora en el S.XXI, la masa crítica, de los grupos de mujeres asociadas deben participar en espacios de poder para romper el androcentrismo.

Propuesta del cuestionario sobre las actividades que normalmente realizan. (Anexo 4)

En la IV Conferencia Mundial sobre las mujeres. Declaración de Beijing, 1995 "... la participación en los procesos de toma de decisiones y el acceso al poder, son fundamentales para el logro de la igualdad, el desarrollo y la paz"

\section{5a Sesión:}

Tema: Es bueno escribir como herramienta de expresión. Ponente: Mujeres escritoras de la asociación.

Momentos de reflexión personal, que nos pueden ofrecer visibilidad y naturalidad a nuestra transición vital. (Freixas ,2014).

\section{6a Sesión:}

Tema: Salud fisiológica.

Ponente: Especialista médica con experiencia sanitaria y con grupos específicos relacionados con la salud.

Salud que nos pueden proporcionar tanto el ejercicio físico, una buena nutrición y la prevención de caídas. La forma de saber en qué momento nos encontramos es posible con el cuestionario. EQ-5B. (Anexo 5).

\section{7ª Sesión:}

Tema: Salud psicológica

Ponente: Psicóloga, con experiencia en adultos.

Cómo podemos "mantener a raya" un posible declive intelectual, que el sentimiento de soledad no nos encierre, que la depresión y el aburrimiento invada nuestro día a día.

\section{REULTADOS}

El planteamiento del taller está distribuido en 7 sesiones, por tanto, sólo podemos conocer el resultado de la primera sesión donde las socias aprobaron el planteamiento presentado sobre el "Envejecimiento activo" También pudimos pasar el cuestionario sobre las responsabilidades de las personas de edad y el resultado queda plasma- 
do en este gráfico que representa el número de respuestas afirmativas de las 19 mujeres que aceptaron dar sus respuestas sobre cada principio.

Fig.4.Rrespuestas positivas a cada principio.

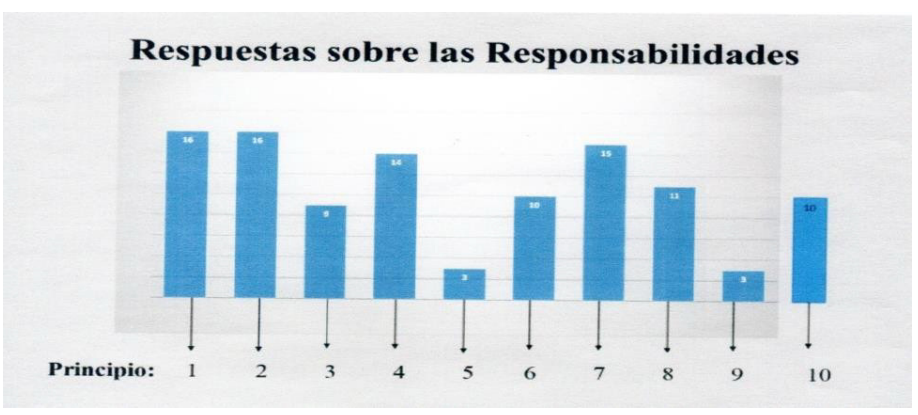

El resto, cuando la pandemia nos lo permita lo pondremos en práctica y podremos observar estudiar el proceso siguiendo el método DAFO en qué momento estamos y hacia dónde dirigir nuestras actividades.

\section{CONCLUSIONES}

La primera conclusión es positiva, las ponentes dispuestas a colaborar, el grupo de mujeres con ganas de empezar y tan sólo esperamos llegar a la conclusión de que el envejecer activamente, nos proporciona fortalezas que nos van a permitir según Fernández-Ballesteros, R. (2002)

Mantener una buena salud y autonomía funcional

Mantener un alto funcionamiento cognitivo

Mantener un alto funcionamiento físico

Mantener un alto compromiso con la vida

\section{REFERENCIAS BIBLIOGRÁFICAS}

Bateson, M.C. (2010). Composing a Further Life: The Age of Active Wisdom. La edificación de una vida más amplia: la edad de la sabiduría activa. Kindle Edition

Cuestionario EQ-5D. Versión en español para US. Pag.2. https://www.ser.es

Delors, J. (1996). La educación encierra un tesoro. UNESCO.

Fernández-Ballesteros, R. (2002). Envejecer bien. Qué es y cómo lograrlo. Vivir con Vitalidad (1) Madrid. Ed. Pirámide

Freixas Farré, A. (2006). Demà més. Dones, vides i temps. Generalitat de Catalunya Barcelona. Col. Quaderns de I'Institut, no 8 .

Freixas Farré, A. (2014). Tan frescas. Las nuevas mujeres del S.XXI. Barcelona. Paidós Col.Contextos.

Hyver, Carlos D. y Gutiérrez Robledo, L.M. (2013). Geriatría. Ed. Manual Moderno .México

Martin Lesende, I, y otros (2007). Envejecimiento activo, la mejor "receta" para prevenir la dependencia. Revista de Española de Geriatría y Gerontología 42 (supl.2).

D0I:10.1016/S011-139X(07)75733-5

Pérez Diaz, J; Abellán García, A; Aceituno Nieto, P; Ramiro Fariñas, D. (2020). Un perfil de las personas mayores en España, 2020. Madrid. Informes Envejecimiento en red n²5, pag.39.

https://envejecimiento.csisc.es/documentos/enred-indicadoresbásicos 2020pdf

Vázquez Resino, M. (2020). Envejecimiento activo en la pandemia del COvid-19.

Reseteando el envejecimiento. https:// WWW.geriatricarea.com/2020/12/15 


\section{ANEXOS}

\section{(1)- LISTADO DE ACTIVIDADES COMPLEMTARIAS}

- Lectura

- Música $\longrightarrow$ componente cultural y emocional

- Teatro $\longrightarrow$ Acción colectiva de comunicación y restablecimiento de la seguridad y autoestima

- Maquetas $\longrightarrow$ Manipulación manual perfeccionando la orientacióny coordinación.

- Xi-Kung $\longrightarrow$ Mantenimiento de las articulaciones con movimientos suaves, coordinados con la respiración.

- Valenciano $\longrightarrow$ El lenguaje como herramienta de comunicación e inclusión.

\section{(2)- LISTADO DE EJECUCIÓN DE LAS RESPONSABILIDADES}

$\square$ Permanecer activas, alertas, capaces, confiadas en el esfuerzo propio y útiles. $\square$ Aprender y aplicar principios sanos de salud física y mental a su propia vida.

$\square$ Hacer planes y prepararse para la vejez y la jubilación y aprovechar la enseñanza encaminada a la alfabetización.

$\square$ Adaptarse a los cambios que con el tiempo puedan ocurrir en las relaciones familiares.

$\square$ Actualizar sus conocimientos y su adiestramiento, si fuera necesario, para aumentar sus posibilidades de obtener empleo si desean participar en la fuerza del trabajo.

$\square$ Poner a disposición de los demás, en particular de las generaciones más jóvenes sus conocimientos, aptitudes y valores espirituales.

$\square$ Participar lo más plenamente posible en la vida cívica de su sociedad.

$\square$ Buscar y desarrollar posibilidades de prestar servicios a los menos afortunados.

$\square$ Nombrar a una tercera persona de su elección para tomar decisiones y representarle.

$\square$ Dar instrucciones de antemano detallando sus decisiones respecto a su salud y bienestar, que incluyan los cuidados que quiera recibir durante su vida y al final de la misma. 
(3)- RELACIONES SOCIALES

\section{¿CÓMO SON SUS RELACIONES SOCIALES?}

\begin{tabular}{|l|l|l|l|l|l|}
\hline $\begin{array}{l}\text { 1. ¿Con qué frecuencia } \\
\text { se encuentra con } \\
\text { personas de su familia } \\
\text { aparte de los } \\
\text { familiares con los que } \\
\text { vive) }\end{array}$ & $\begin{array}{l}\text { Varias } \\
\text { veces } \\
\text { a la } \\
\text { semana }\end{array}$ & Semanalmente & Quincenalmente & $\begin{array}{l}\text { Cada } \\
\text { mes }\end{array}$ & $\begin{array}{l}\text { Menos de } \\
\text { una vez al } \\
\text { mes }\end{array}$ \\
\hline $\begin{array}{l}\text { 2. ¿Con qué frecuencia } \\
\text { se reúne con amigos? }\end{array}$ & $\begin{array}{l}\text { Varias } \\
\text { veces } \\
\text { a la } \\
\text { semana }\end{array}$ & Semanalmente & Quincenalmente & $\begin{array}{l}\text { Cada } \\
\text { mes }\end{array}$ & $\begin{array}{l}\text { Menos de } \\
\text { una vez } \\
\text { al mes }\end{array}$ \\
\hline $\begin{array}{l}\text { 3. ¿Con qué frecuencia } \\
\text { charla con sus vecinos? }\end{array}$ & $\begin{array}{l}\text { Varias } \\
\text { veces } \\
\text { a la } \\
\text { semana }\end{array}$ & Semanalmente & Quincenalmente & $\begin{array}{l}\text { Cada } \\
\text { mes }\end{array}$ & $\begin{array}{l}\text { Menos de } \\
\text { una vez } \\
\text { al mes }\end{array}$ \\
\hline $\begin{array}{l}\text { 4. ¿Se siente satisfecha } \\
\text { con sus relaciones con } \\
\text { su } \\
\text { familia? }\end{array}$ & Nada & Algo & Bastante & Mucho & Muchísimo \\
\hline $\begin{array}{l}\text { 5. ¿Se siente satisfecha } \\
\text { con sus relaciones con } \\
\text { sus amigos? }\end{array}$ & Nada & Algo & Bastante & Mucho & Muchísimo \\
\hline $\begin{array}{l}\text { 6. ¿Se siente satisfecha } \\
\text { con sus relaciones con } \\
\text { sus vecinos y } \\
\text { conocidos? }\end{array}$ & Nada & Algo & Bastante & Mucho & Muchísimo \\
\hline $\begin{array}{l}\text { 7. ¿Con qué frecuencia } \\
\text { se siente sola? }\end{array}$ & Nada & Algo & Bastante & & \\
\hline
\end{tabular}

"Vivir con vitalidad (1). Envejecer bien. ¿Qué es y cómo lograrlo?”. Fernández-Ballesteros, R. (2002)

(4). ¿QUÉ ACTIVIDADES HACES? 
A continuación, se ha listado un conjunto de actividades, por favor, ponga una cruz en la casilla correspondiente a la frecuencia con la que usted ha realizado es actividad en el último mes:

Nada (1), Algo (2), Bastante (3), Mucho (4), Muchísimo (5)

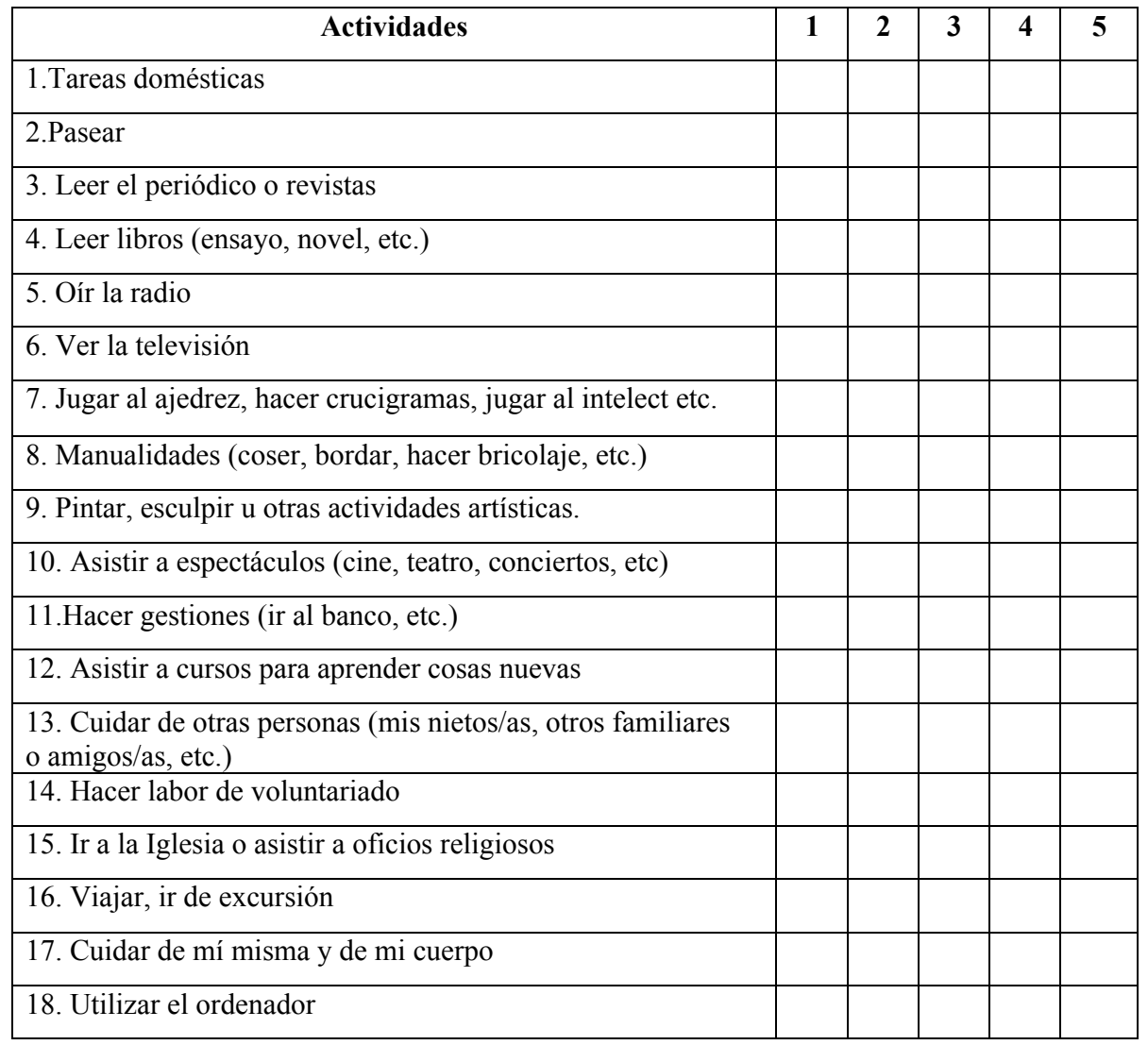

"Vivir con vitalidad (1). Envejecer bien. ¿Qué es y cómo lograrlo?”. Fernández-Ballesteros, R. (2002)

(5)-CUESTIONARIO DE SALUD. EQ-5D 
Marque con una cruz como esta $₫$ la afirmación en cada sección que describa mejor su estado de salud en el día de hoy.

\section{Movilidad}

No tengo problemas para caminar

Tengo algunos problemas para caminar

Tengo que estar en la cama

\section{Cuidado-Personal}

No tengo problemas con el cuidado personal

Tengo algunos problemas para lavarme o vestirme solo

Soy incapaz de lavarme o vestirme solo

Actividades de Todos los Días (ej, trabajar, estudiar, hacer tareas domésticas, actividades familiares

o realizadas durante el tiempo libre)

No tengo problemas para realizar mis actividades

de todos los días

Tengo algunos problemas para realizar mis actividades

de todos los días

Soy incapaz de realizar mis actividades de todos los días

\section{Dolor/Malestar}

No tengo dolor ni malestar

Tengo moderado dolor o malestar

Tengo mucho dolor o malestar

\section{Ansiedad/Depresión}

No estoy ansioso/a ni deprimido/a

Estoy moderadamente ansioso/a o deprimido/a

Estoy muy ansiosola o deprimido/a 
Como las respuestas son anónimas, la información personal que le pedimos a continuación nos ayudará a valorar mejor las respuestas que nos ha dado.

1. ¿Tiene usted experiencia en enfermedades graves? (conteste a las tres situaciones)

$\begin{array}{lll}\text { en usted mismo } & \text { Si } & \text { No } \\ \text { en su familia } & \square & \square \\ \text { en el cuidado de otros } & \square & \square \\ \end{array}$

POR FAVOR PONGA UNA CRUZ EN LA CA.JITA

2. ¿Cuántos años tiene?

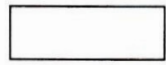

3. Es usted:

$\begin{array}{cc}\text { Varón } & \text { Mujer } \\ \square & \square\end{array}$

POR FAVOR PONGA UNA CRUZ EN LA CA.ITA

4. Es usted:

fumador
ex-fumador
nunca ha fumado

$\square$

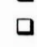

POR FAVOR PONGA UNA CRUZ EN LA CAJITA

5. ¿Trabaja o ha trabajado en servicios de salud o sociales?

Si No

口

POR FAVOR PONGA UNA CRUZ EN LA CA.JITA

Si ha contestado sí, en calidad de qué?.

6. ¿Cuál es su principal actividad actual?

empleado o trabaja para si mismo
retirado o jubilado
tareas domésticas
estudiante
buscando trabajo
otros (por favor especifique)

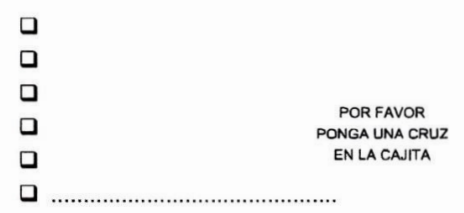

7. ¿Nivel de estudios completados?

Leer y escribir

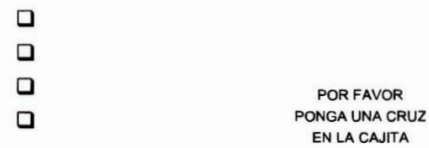

8. Si conoce su código postal, por favor escribalo aquí:

Secundaria, vocacional

Universidad

EN LA CAJITA 
